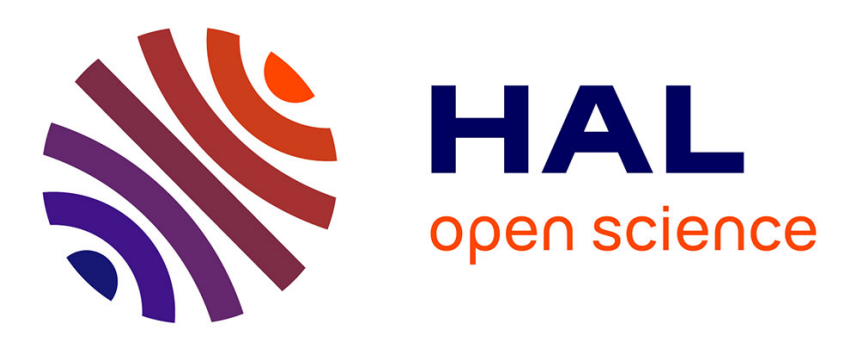

\title{
An Event-Controlled Online Trajectory Generator Based on the Human-Robot Interaction Force Processing
}

\author{
Sarra Jlassi, Sami Tliba, Yacine Chitour
}

\section{To cite this version:}

Sarra Jlassi, Sami Tliba, Yacine Chitour. An Event-Controlled Online Trajectory Generator Based on the Human-Robot Interaction Force Processing. Industrial Robot: An International Journal, 2014, 41 (1), pp.15-25. 10.1108/IR-01-2013-317 . hal-00861854

\section{HAL Id: hal-00861854 https://hal.science/hal-00861854}

Submitted on 13 Sep 2013

HAL is a multi-disciplinary open access archive for the deposit and dissemination of scientific research documents, whether they are published or not. The documents may come from teaching and research institutions in France or abroad, or from public or private research centers.
L'archive ouverte pluridisciplinaire HAL, est destinée au dépôt et à la diffusion de documents scientifiques de niveau recherche, publiés ou non, émanant des établissements d'enseignement et de recherche français ou étrangers, des laboratoires publics ou privés. 


\section{An Event-Controlled Online Trajectory Generator Based on the Human-Robot Interaction Force Processing}

Authors: Sarra Jlassi, Sami Tliba and Yacine Chitour

S. Jlassi, S. Tliba and Y. Chitour are with Laboratoire des Signaux et Systèmes UMR8506, Univ Paris-Sud, CNRS, SUPELEC, 3 rue Joliot Curie, 91192 Gif-sur-Yvette, France (corresponding author e-mail: sami.tliba@lss.supelec.fr).

Université Paris-Sud XI,

U.F.R. des Sciences d'Orsay

Layout by Sami TLIBA, (c) July 2013
UMR : Laboratoire des Signaux \& Systèmes

Projet : Robotic comanipulation

Type : Preprint to "Industrial Robot: an International Journal" 


\begin{abstract}
The problem of robotic co-manipulation is often addressed using impedance control based methods where we seek to establish a mathematical relation between the velocity of the human-robot interaction point and the force applied by the human operator at this point. This paper addresses the problem of co-manipulation for handling tasks seen as a constrained optimal control problem. The proposed point of view relies on the implementation of a specific online trajectory generator (OTG) associated to a kinematic feedback loop. This OTG is designed so as to translate the human operator intentions to ideal trajectories that the robot must follow. It works as an automaton with two states of motion whose transitions are controlled by comparing the magnitude of the force to an adjustable threshold, in order to enable the operator to keep authority over the robot's states of motion. To ensure the smoothness of the interaction, we propose to generate a velocity profile collinear to the force applied at the interaction point. The feedback control loop is then used to satisfy the requirements of stability and of trajectory tracking to guarantee assistance and operator security. The overall strategy is applied to the penducobot problem.
\end{abstract}

keywords: Co-manipulation for handling tasks, online trajectory generator, underactuated robots

\title{
1 Introduction
}

Robotics research is shifting its focus from industrial applications to human centered ones. The ultimate goal is to reduce fatigue, improve the motions' precision, amplify the power and enhance the daily life. Typical application modes of these robots include entertainment, cooperation as in [Lawitzky et al., 2010], teleoperation (e.g. [Itoh et al., 2000]) etc. In cooperation mode, the sought behavior is typically that of a master-slave relationship [Kazerooni and Guo, 1993]. In [Wannasuphoprasit et al., 1997]; [Colgate et al., 1996], robotic systems manipulating objects in collaboration with a human operator (HO) are considered. The HO expresses his intention through a desired motion and the robot has to carry out the task by bringing the necessary efforts and/or adapt its impedance. Such robots are useful in automotive industry as described in [Akella et al., 1999] or also in the case of a truck driver who has to replace a defective but heavy tire. This problem could be tackled with a robot that remains under the authority of the $\mathrm{HO}$ who interacts directly with the robot. The robot assists the human during his motion while relieving him from the important effort without any human perceptible resistive forces like inertia, friction etc. Humans are skilled at complex tasks and are able to react very flexibly to unknown situations. Industrial robots are very strong, fast and accurate. It is important to note the difference between the frequencies with which a $\mathrm{HO}$ and the robot can perform their tasks. Studies of [Miyhoshi and Murata, 2000] have shown that a human can perform a task with a frequency of up to $6 \mathrm{~Hz}$, which is much slower than the typical sampling frequency used in a robotic control scheme. During physical human-robot interaction (HRI), the skills of both should be combined and the resulting cooperative motion should be truly intuitive and should not restrict in any way the human motion. This is often referred to as transparency. There are still many challenges on the road to achieving an efficient physical HRI [Santis et al., 2008]. These challenges are mainly related to safety (stability) and to developing robot capabilities. One of the most commonly used control methods in HRI is the impedance/admittance control [Akella et al., 1999, Lamy et al., 2010]. Its purpose is to reduce the mechanical interaction point (IP) impedance so as to make it equal to that of HO [Buerger, 2005]. In the context of co-manipulation for handling tasks, usual impedance control based methods have few drawbacks, particularly noticeable during the starting and the stopping phases. As explained in [Duchaine and Gosselin, 2011], the use of a fixed virtual damping can lead to an inefficient co-manipulation. In order to provide a better HRI, it was proposed to continuously adapt the virtual damping coefficient as function of the human "intention". This approach was performed using the time derivative of the measured force, for which the measurement noises can cause problems with the numerical differentiation (dividing by zero, etc.). 
Our approach stands out for the nature of the problem to be tackled, viz. heavy load handling tasks, and for its vision on the co-manipulation. It is based on the implementation of two main ingredients. The first one lies in the online generation of an appropriate trajectory of the IP located at the end-effector and describing the HO intention. The other consists in the design of a control structure allowing a good tracking of the generated trajectory. The force applied by the HO is the only physically exchanged signal showing the robot how to move according to his willingness. Indeed, this force gives the information about the desired displacement direction of the end-effector. But, this direction should necessarily be collinear to the IP velocity. In [Jlassi et al., 2012], the idea of maintaining to the maximum the collinearity of the end-effector velocity and the interaction force was proposed to concur in achieving a transparent co-manipulation. The required force to accomplish the comanipulation task is supplied by the robot's actuators, controlled in order to ensure a good trajectory tracking. Furthermore, for sake of safety, we consider that the displacement velocity should not entirely depend on HO's desire but must be conform to a given velocity template. The literature provides a very rich set of approaches and algorithms of trajectory generation for industrial manipulators [Kröger, 2010]. In this paper, we address the case of force sensor-based trajectory generation. The interaction force processing should contribute to both interpreting the triggering times of movements/immobility and in commanding how the robot has to move or stop, while leaving the final position under the human authority. The trajectory is calculated online, i.e. each desired position is updated during every control cycle because the input values based on the HO force (intensity and direction) may change unpredictably.

The remainder of this paper is organized as follows: After an analysis in Section 2, of the co-manipulation requirements and their consequences on the problem statement, the control strategy based on the criterion of collinearity between the interaction force and the end-effector velocity is presented in Section 3 . The description of the proposed OTG is developed in Subsection 3.3. The controller design method is explained in Subsection 3.4. The OTG algorithm is applied and discussed in detail in Subsection 4.3, through the demonstrative example of the penducobot presented in Section 4. Finally, some conclusions, perspectives and future researches are given in Section 5 .

\section{Co-manipulation requirements and trade-off}

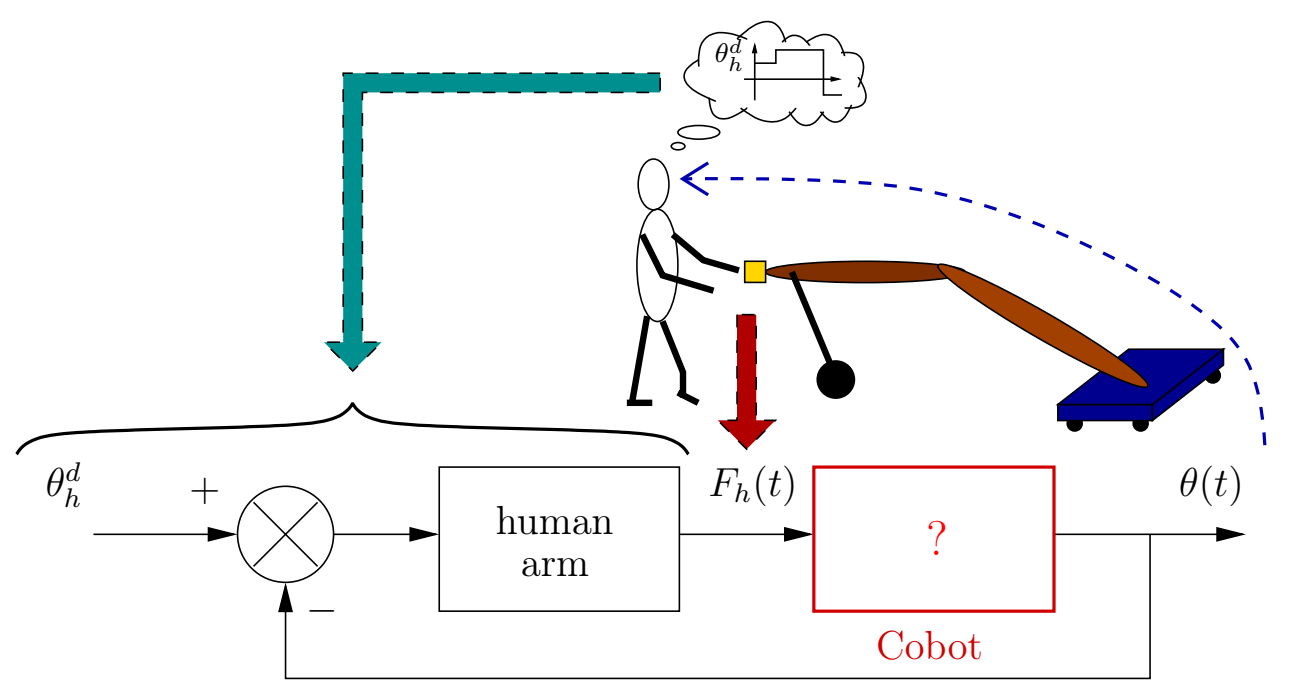

Figure 1: Concept of co-manipulation 
This work focuses on heavy load handling tasks characterized by having motion with moderate velocities. A potential application field for the strategy proposed in this paper is the manufacturing industry such as construction industry and assembly where material handling requires considerable efforts. The main purpose is reducing these efforts, decreasing the consumed time and the costs of these activities. The mechanical links of the considered robots are all of revolute type. A pendulous load is linked to the last arm (end-effector) with a passive joint (unactuated), which is also the arm that interacts with the HO. A force sensor measures the interaction force and gives its corresponding coordinates along each direction in the end-effector frame. Its localization should be chosen to suit the co-manipulation task. Since the HO will typically interact with the robot end-effector and not directly with the load, the force sensor could be mounted on the end-effector of the robot. The idea behind this choice of sensor position is to distinguish between the human applied force and the disturbance caused by the mass (load) uncertainty. In other words, the resultant force of the load seen as a disturbance should not be detected by the sensor. However for sake of robustness it should be compensated by the position control loop. It is assumed that all the robot actuators are torque controlled and that the measures of position and velocity are all accessible. The human-robot co-manipulation problem is considered here as the achievement of a master-slave relationship, for which the HO decides when and where the robot moves. In other words, the $\mathrm{HO}$ contributes in the co-manipulation process by making a visual feedback loop of the current robot configuration with the desired one that he has in mind, as described schematically by Figure 1. This problem consists in guaranteeing the safety of the $\mathrm{HO}$ while manipulating the robot from its end-effector and driving it to the desired position without having to exert the required force to move the load, or even to perceive the resistive forces due to the robot dynamics such as its strong inertia, joints friction etc. Hence, this problem consists in designing the block named Cobot, in a way that it satisfies the requirements below when running.

a) Assistance: The HO drives the end-effector of the robot which accordingly ensures the provision of the necessary power to move the handled object or to maintain it in a desired equilibrium position.

b) Transparency: During HRI, the resulting cooperative motion should not restrict in any way the operator's willingness to move the robot as he would like. Thus, the operator should not perceive any disturbing effects due to the robot resistive forces.

c) Human safety: For physical HRI, safety is of utmost importance due to the potential risk that may cause a relatively powerful robot. From the control standpoint, it is possible to increase this level of safety by guaranteeing that the robot will never exhibit any unacceptable behaviors like instability, too fast motion etc.

The level of importance of each requirement depends on the expected purpose of co-manipulation. For the case of heavy load handling task, ensuring the maximum possible of transparency may cause a serious danger for the operator. Thus, a trade-off between transparency and security appears necessary. Here, this trade-off is in favor of security. Our interpretation of transparency requirement is translated as control objectives for which velocity of the IP should remain collinear to the force exerted by the HO and should be proportional to the magnitude of this force. Notice that exerting a force is the only way for the HO to transmit the information that contains intrinsically the desired direction of displacement and this direction is given by the velocity vector at each instant. In consequence, the measured force should serve in generating a trajectory for the end-effector velocity that this latter has to track. Moreover, the trajectory to track must be generated online by an appropriate real-time processing of this information. To cope with safety requirement, the kinetic energy stored by the robot during the motion has to be limited by imposing either a profile or a template to the desired trajectory for the end-effector velocity. A template basically consists in imposing an upper bound on the magnitude of this velocity. But in our case, this also requires imposing an upper bound on its successive derivatives in order to filter the tremors introduced by the exerted force. On the other hand, the choice of 
a profile, which consists in using parameterized functions, is easier to implement in an OTG and allows to cope with the problem of tremors. This latter approach will thus be adopted in the sequel. The assistance requirement will be satisfied once the trajectory generation and its tracking will be properly achieved. Then, generation and tracking represent the main challenges that will be tackled thereafter, a global problem that can be formulated as a constraint optimal control problem.

\section{Control strategy}

\subsection{Working frame and notations}

Without loss of generality, our development will focus on the case of planar robots. Let $\vec{X} \in \mathbb{R}^{2}$ be the position vector of the end-effector.

Let $n$ be the total number of the Degrees Of Freedom (DOFs) and $q \in \mathbb{R}^{n}$ the vectors of the generalized joints displacements, i.e.the active and passive joints noted $q_{a}$ and $q_{p} \in \mathbb{R}$ respectively. The relationship between the instantaneous velocity of the end-effector and the velocities of the active joints is given by

$$
\dot{\vec{X}}=J\left(q^{a}\right) \dot{q}^{a},
$$

where $J\left(q^{a}\right)$ is the manipulator Jacobian. The well-known dynamical model of the robot is given by

$$
M(q) \ddot{q}+C(q, \dot{q}) \dot{q}+g(q)=U+W_{f}+W_{H O} .
$$

The control vector is $U:=\left[\begin{array}{ll}\tau^{T} & 0\end{array}\right]^{T} \in \mathbb{R}^{n}$, where $\tau \in \mathbb{R}^{n-1}$ is the vector of applied torques to the active joints and 0 is the scalar null torque applied to the passive joint. The vector of HRI torques is given by $W_{H O}:=J\left(q^{a}\right)^{T} \overrightarrow{F_{h}}$, where $\overrightarrow{F_{h}} \in \mathbb{R}^{2}$ is the interaction force exerted at the end-effector. This force vector is characterized by its Euclidian norm noted $F_{h}=\left\|\overrightarrow{F_{h}}\right\|$ and its orientation, defined by the unitary vector, noted $\overrightarrow{U_{\theta_{h}}}$, where $\theta_{h}$ is the angle formed by the interaction force $\overrightarrow{F_{h}}$ and a unitary vector of the fixed frame. Denote by $\overrightarrow{V_{\theta_{h}}}$ the unitary vector, orthogonal and positively oriented to $\overrightarrow{U_{\theta_{h}}}$. The terms $M(q) \in \mathbb{R}^{n \times n}, C(q, \dot{q}) \dot{q} \in \mathbb{R}^{n}$, $g(q) \in \mathbb{R}^{n}$ and $W_{f} \in \mathbb{R}^{n}$ are employed to denote the inertia matrix (symmetric and positive definite), the vector containing the centrifugal and Coriolis forces, the vector of gravitational forces and the vector of friction forces respectively.

\subsection{Collinearity between force and end-effector velocity}

As mentioned previously, maintaining to the maximum the collinearity of the end-effector velocity and the interaction force while having the same direction, should concur in achieving a transparent co-manipulation. The co-manipulation expected requirements should lead the end-effector velocity and the interaction force to satisfy a relationship like

$$
\dot{\vec{X}}=\chi\left(q, \dot{q}, \overrightarrow{F_{h}}\right)
$$

under the constraints of zero cross-product $\dot{\vec{X}} \times \overrightarrow{F_{h}}=0$ and of positive scalar product $\left\langle\dot{\vec{X}}, \overrightarrow{F_{h}}\right\rangle>0$, where $\chi\left(q, \dot{q}, \overrightarrow{F_{h}}\right)$ is a mathematical operator that can be assimilated to an admittance relationship, but which expression is non-trivial and certainly hard to express analytically, because it has to satisfy all the requirements in an optimal manner. In particular, $\chi\left(q, \dot{q}, \overrightarrow{F_{h}}\right)$ should be able to cope with discrete events such as the human decision of starting or stopping the robot motion, and to maintain a stable robot equilibrium position, robust to exogenous disturbances. Usually, this operator is described in frequency domain and taken as a linear admittance. But it is clear that a linear admittance cannot simply cope with start and stop phases. It was shown 
in [Ikeura et al., 1994] that damping parameters of the linear admittance are the predominant coefficients that allow setting the acceleration/deceleration features in the context of HRI. If the damping is set to a low value, then, when the $\mathrm{HO}$ wants to stop the robot, it will be so compliant that it could collapse, which would represent a serious danger. Otherwise, the robot will be hard to manipulate and thereby restricts the human motion. The use of a variable damping adjusted according to the intention of the HO provides a solution but it has a drawback related to the necessity of computing the force derivative, as explained in [Duchaine and Gosselin, 2011].

In this paper, the seeking of the operator $\chi\left(q, \dot{q}, \overrightarrow{F_{h}}\right)$ is addressed in a suboptimal way, through the two steps design of an OTG and a controller, following the method described thereafter.

\subsection{Online Trajectory Generator}

\section{a) Force intensity processing}

Define a force threshold, noted $f_{t h}$, that allows distinguishing the intention to move the robot if the interaction force amplitude is greater than $f_{t h}$, and the willingness to stop it if the force is lower. Let $h_{r}$ and $h_{f}$ be the logical quantities that switch to 1 when the force measurement crosses the threshold $f_{\text {th }}$, with a rising and falling edge respectively. When $h_{r}$ switch to 1 , it means that the robot enters into a state of motion, noted $\Sigma_{\text {motion }}$, and it remains there. It is therefore an event from which two phases follow each other, a transient phase corresponding to the motion startup, noted $\phi_{\text {rise }}$, and a phase of established motion, $\phi_{\text {cruise }}$. When $h_{f}$ switch to 1 , it means that the robot enters into a state of immobility, noted $\Sigma_{i m m}$., and stops at the desired position. It is also an event from which two phases follow each other, a transient phase corresponding to the decelerating motion until the stop, noted $\phi_{\text {fall }}$, and another one in which the robot configuration is maintained in a equilibrium position, $\phi_{\text {stop }}$. To realize a more natural motion, the transient phases and the one of established motion should be customizable. Let $\varepsilon_{f}$ be the duration of $\phi_{\text {fall }}$ and $\varepsilon_{r}$ that of $\phi_{\text {rise. These settings depend }}$ on the nature of the manipulated object, its environment and the operator's capabilities. The customization of the transient part should allow adjusting the reaction time to match the minimum effort that can apply the operator, according to his abilities. The setting of the established motion phase should be compatible with the constraint imposed on the IP's velocity, i.e.with a velocity of constant and moderate amplitude, noted $V_{0}$. Only the duration control of $\phi_{\text {stop }}$ and $\phi_{\text {cruise }}$ is left to the HO's decision. Hence, the interaction force processing allows the identification of the initiation moment of the rising or the falling phase (denoted $t_{0}$ ), making thus the OTG event-controlled with two discrete states governed by the automaton of Figure 2. This moment is captured by comparing continuously the magnitude of this force with $f_{t h}$. The sequencing of each phase always follows the same closed periodical cycle as depicted in Figure 3.

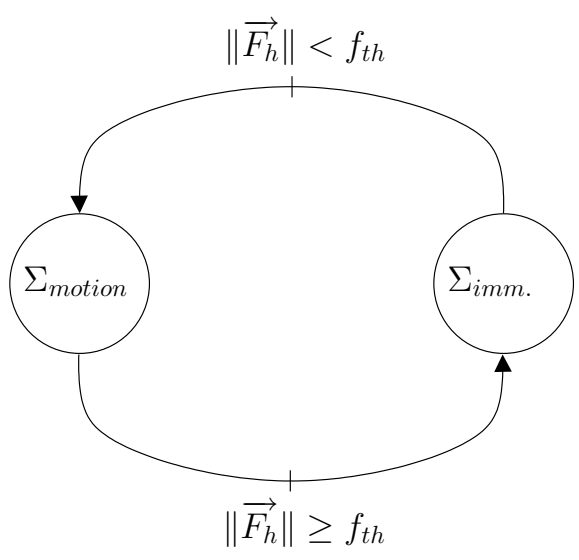

Figure 2: Automaton governing the motion states

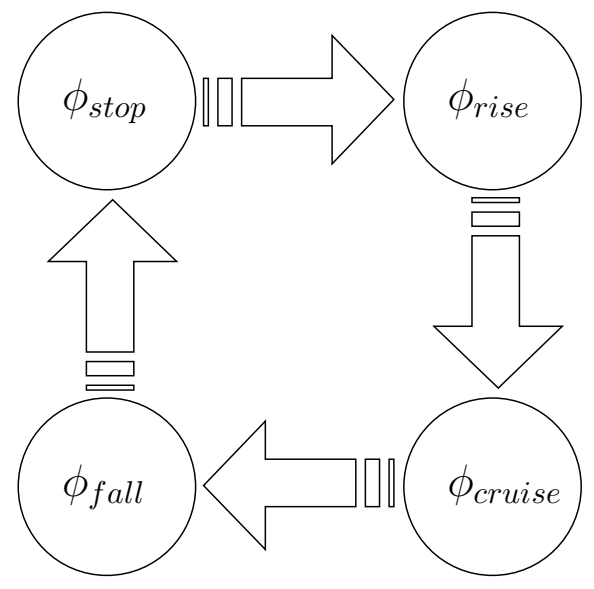

Figure 3: Periodical cycle of phases 


\section{b) Low-jerk velocity profile}

The role of the OTG is to convert the real-time processing of the interaction force into displacement instructions of the IP, that depend on the phase in which is the HRI. These instructions should rely on a specific velocity profile, noted $V_{p}(t)$, that meets the expected requirements on the norm of the desired velocity at the IP (noted $\dot{\overrightarrow{X_{d}}}$ ), viz safety, transparency and fluidity of movement interaction. A clear understanding of the human arm motions, as studied extensively by authors in [Tee et al., 2010], should contribute to a better consideration of the co-manipulation requirements in the definition of the trajectories to track. However, for simplicity and conciseness reasons of this paper, we made the choice of not including the human arm's dynamic to model the HRI in terms of force and common trajectories, whereas it should be included for a more rigorous and complete study. In return, a simpler representation of the HRI is considered. Since natural trajectories are required for a safe, smooth and comfortable interaction between the HO, the robot and the hanged load, we adopted the minimal-jerk trajectories to represent the HRI. Indeed, smoothness can be quantified as a function of jerk as reported in [Flash and Hogans, 1985]. It is now well established and experimentally demonstrated that limiting jerk is important for reducing the robot attrition as well as for improving fast and accurate tracking [Piazzi and Visioli, 2000], [Craig, 1989], [Constantinescu and Croft, 2000]. Moreover, it leads to a smoother control of the actuators, which reduces the excitation of the manipulator's fast dynamics such as vibrating modes. This consequently reduces danger for the HO, wear of the actuators, etc. To gather these specifications, the proposed low-jerk time function $V_{p}(t)$ imposed to $\left\|\dot{\overrightarrow{X_{d}}}\right\|$ and satisfying each phase's requirement is given by a piecewise defined polynomial based on the following quitinc polynomial

$$
V_{p}(t)=V_{i}+\left(V_{f}-V_{i}\right)\left(10\left(\frac{t-t_{0}}{T}\right)^{3}-15\left(\frac{t-t_{0}}{T}\right)^{4}+6\left(\frac{t-t_{0}}{T}\right)^{5}\right)
$$

where $T, V_{i}$ and $V_{f}$ are parameters governed by the current HRI phase, as described in Table 1.

\begin{tabular}{|c|c|l|}
\hline Phase & Parameters setting & Velocity profile expression \\
\hline$\phi_{\text {stop }}$ & $T \neq 0, V_{i}=0, V_{f}=0$ & $V_{p}(t)=0$ \\
\hline$\phi_{\text {rise }}$ & $T=\varepsilon_{r}, V_{i}=0, V_{f}=V_{0}$ & $V_{p}(t)=V_{0}\left(10\left(\frac{t-t_{0}}{\varepsilon_{r}}\right)^{3}-15\left(\frac{t-t_{0}}{\varepsilon_{r}}\right)^{4}+6\left(\frac{t-t_{0}}{\varepsilon_{r}}\right)^{5}\right)$ \\
\hline$\phi_{\text {cruise }}$ & $T \neq 0, V_{i}=0, V_{f}=V_{0}$ & $V_{p}(t)=V_{0}$ \\
\hline$\phi_{\text {fall }}$ & $T=\varepsilon_{f}, V_{i}=V_{0}, V_{f}=0$ & $V_{p}(t)=V_{0}\left(1-10\left(\frac{t-t_{0}}{\varepsilon_{f}}\right)^{3}+15\left(\frac{t-t_{0}}{\varepsilon_{f}}\right)^{4}-6\left(\frac{t-t_{0}}{\varepsilon_{f}}\right)^{5}\right)$ \\
\hline
\end{tabular}

Table 1: Phase dependent velocity profile expression

\section{c) Force direction processing}

The desired velocity $\dot{\overrightarrow{X_{d}}}$ of the IP should remain collinear at every moment to the force exerted by the HO while having the same direction. Thus the angle formed by the IP's desired velocity and the interaction force $\left(\overrightarrow{X_{d}}, \overrightarrow{F_{h}}\right)$ should be zero during the co-manipulation task, which is equivalent to ensuring $\dot{\vec{X}} \times \overrightarrow{F_{h}}=0$ with $\left\langle\dot{\vec{X}}, \overrightarrow{F_{h}}\right\rangle>0$. In other words, $\dot{\overrightarrow{X_{d}}}$ should have the same orientation angle than $\overrightarrow{F_{h}}$, namely $\theta_{h}$. So, the direction to track, 
given by the unitary vector $\overrightarrow{U_{\theta_{h}}}$, has to be extracted from the measured force $\overrightarrow{F_{h}}$. The force sensor delivers the coordinates of $\overrightarrow{F_{h}}$ in the end-effector frame. A simple use of rotational transformations gives the corresponding coordinates in task space frame, $\overrightarrow{F_{h}}=\left[\begin{array}{ll}F_{x} & F_{y}\end{array}\right]^{T}$. Then, the orientation angle is given by $\theta_{h}=\tan ^{-1}\left(F_{y} / F_{x}\right)$. In consequence, once the desired norm of $\dot{\overrightarrow{X_{d}}}$ computed as indicated in Paragraph b), the coordinates of $\dot{\overrightarrow{X_{d}}}$ in task-space frame are easily derived by the relation: $\dot{\overrightarrow{X_{d}}}=V_{p} \overrightarrow{U_{\theta_{h}}}$. The desired position $\overrightarrow{X_{d}}$ is obtained by a coordinate numerical integration of $\dot{X_{d}}$, using for example an Euler explicit method, whereas the corresponding acceleration $\ddot{\overrightarrow{X_{d}}}$ is deduced through basic numerical differentiation. All those vectors are used in an Inverse Kinematic Model component (IKM) [Spong et al., 2006] in order to compute the relevant variables needed in our control structure, such as joint positions, velocities and accelerations.

\section{d) Recursive algorithm}

Because the human may change his desired motion unpredictably (a sudden presence of an obstacle, an unexpected change of destination etc), the real-time processing of the interaction force requires to store the starting and stopping moments of the robot's motion in memory cells, together with the values of the corresponding state i.e.position, velocity etc. Since the desired motion signals $\left(\overrightarrow{X_{d}}, \dot{\overrightarrow{X_{d}}}\right.$ and $\left.\ddot{\overrightarrow{X_{d}}}\right)$ are generated in real-time, the information needed at the next computation cycle must be stored at each instant to allow. The algorithm for generation of these guidelines is necessarily recursive and can be represented by the block-scheme in Figure 4 .

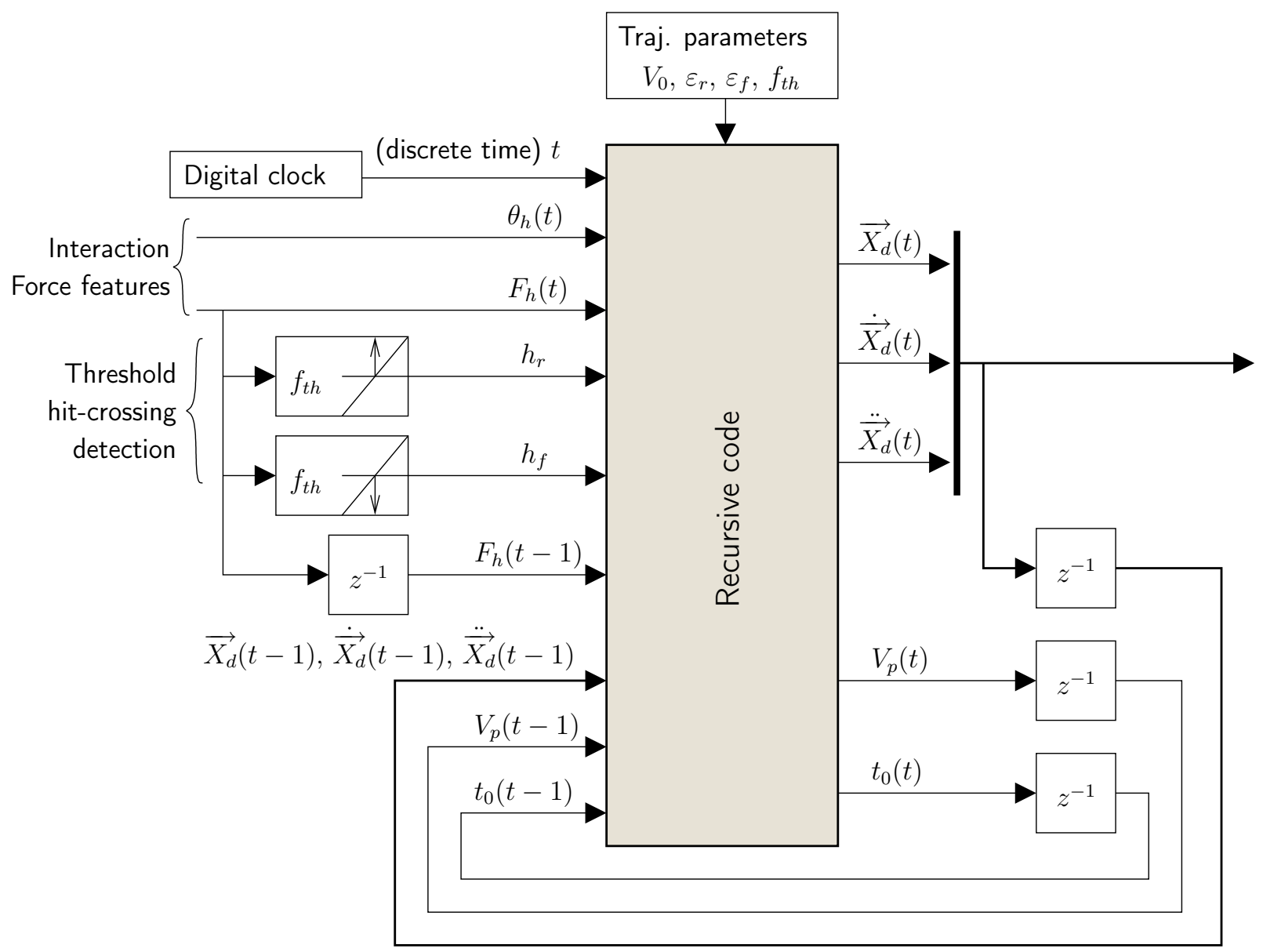

Figure 4: Block-scheme of the recursive algorithm for generating the desired trajectory 


\subsection{Controller design procedure}

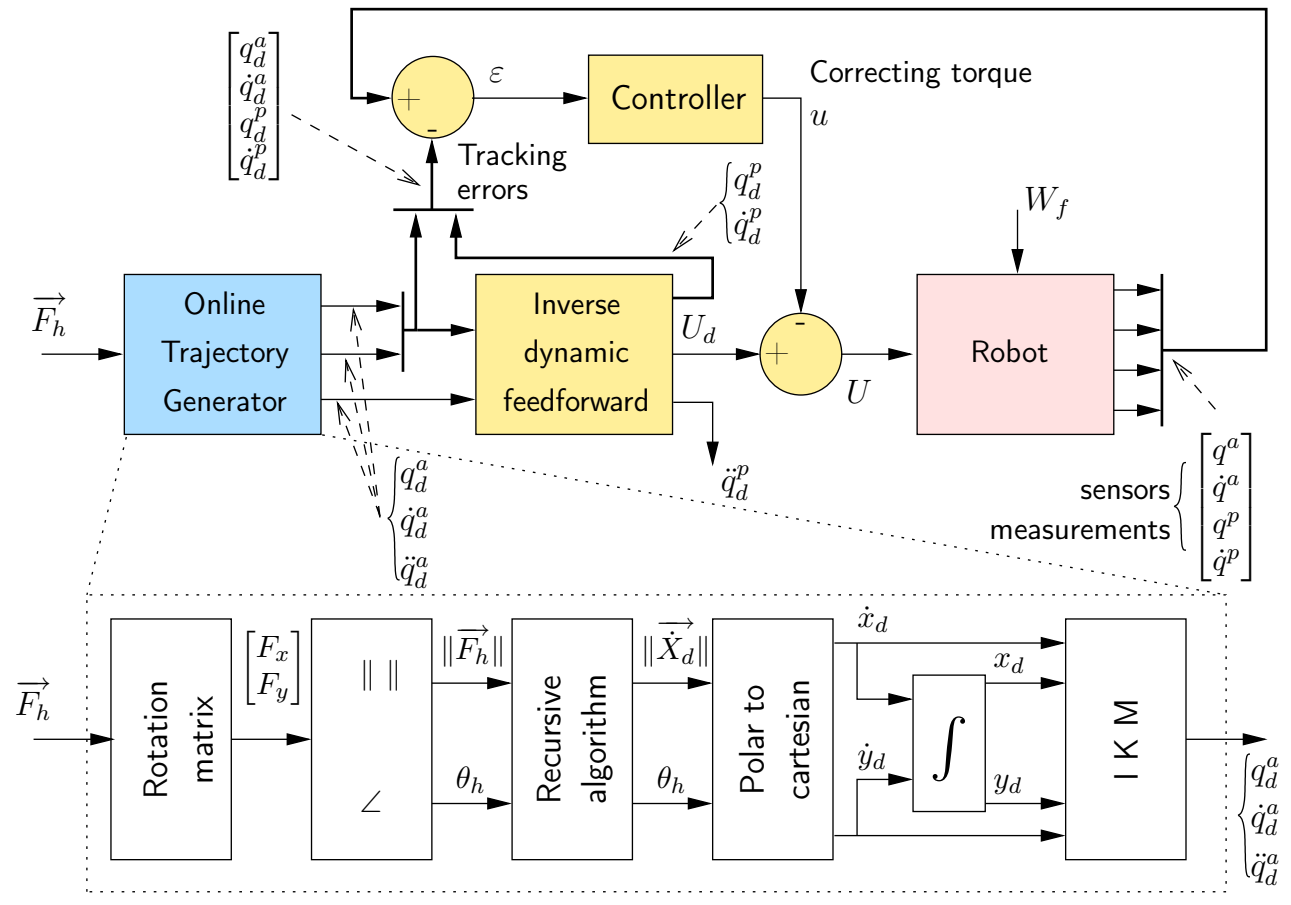

Figure 5: Control structure of planar robot with one passive DOF

\section{a) Inverse dynamic based control}

The OTG component described in Subsection 3.1 provides the trajectory for the active DOFs, noted $q_{d}^{a}$. Since the actuators are torque-controlled, it is then necessary to generate the desired torque, noted $U_{d}$, consistent with $q_{d}^{a}$. Moreover, the considered robot has one unactuated joint. Thus, the control strategy should also cope with the stabilization problem of the passive joint. For this purpose, the generation of an appropriate trajectory for this DOF, noted $q_{d}^{p}$, is also considered in order to take into account the trajectory tracking error of this DOF in the control. In the proposed work, both $U_{d}$ and $q_{d}^{p}$ are obtained by the numerical inversion of the robot dynamical model in (2), so as to be consistent with it when $q^{a} \equiv q_{d}^{a}$. This is implemented in the component named "Inverse Dynamic Feedforward" of the control structure depicted in Figure 5.

\section{b) Controller design}

To cope with the uncertainties (due to unknown initial conditions, parameters uncertainties etc) that may deteriorate the results of the model inversion, the robotic system is inserted in a feedback loop containing a controller designed to make the tracking error vanish. Let $\tilde{q}:=q-q_{d}$ and $\dot{\tilde{q}}:=\dot{q}-\dot{q}_{d}$ be the position and velocity error respectively, where $q_{d}=\left[\begin{array}{ll}q_{d}^{a T} & q_{d}^{p T}\end{array}\right]^{T}$. The controller should be designed in order to ensure an asymptotic convergence of the DOFs toward the desired generated trajectories, i.e. $\lim _{t \rightarrow \infty} \tilde{q}(t)=0$. The model inversion based control law $U_{d}$ is summed to a torque correction term $u$, corresponding to the output of the sought controller that should ensure the tracking requirement:

$$
U:=u+U_{d}
$$

Despite the fact that any kind of controller could be considered in this work, for sakes of simplicity, the proposed approach is limited to the synthesis of a linear controller based on the results of the following theorem [Coron, 2007]. 
Theorem 1 Consider the nonlinear system

$$
\dot{x}=f(x)+g(x) U
$$

where $f$ and $g$ are smooth vector fields, $x \in \mathbb{R}^{2 n}$ is the state of the system and $U \in \mathbb{R}^{n}$ is the control vector.

- If the linearized system around the equilibrium point $P_{0}:=\left(x_{0}, U_{0}\right) \in \mathbb{R}^{2 n} \times \mathbb{R}^{n}$ is controllable, then it is stabilizable and the non-linear system (6) is locally controllable around $x_{0}$ and locally stabilizable with the same feedback control law in a neighborhood of $P_{0}$.

- Let $\left(x_{d}(t), U_{d}(t)\right)$ be the pair of the desired trajectory $x_{d} \in \mathbb{R}^{2 n}$ and the corresponding control $U_{d} \in \mathbb{R}^{n}$, satisfying (6) for all $t \geq 0$. Suppose that $f(0)=0$. Let $A:=\left.\frac{\partial f}{\partial x}\right|_{x=0}$ and $B:=g(0)$. Assume that the pair $(A, B)$ is completely controllable. Let $K \in \mathbb{R}^{n \times 2 n}$ be a state feedback gain matrix such that $A+B K$ is Hurwitz.

$$
U=K\left(x-x_{d}\right)+U_{d}
$$

Then, the control law (7) insures locally an exponential convergence of $(x(t), U(t))$ toward $\left(x_{d}(t), U_{d}(t)\right)$.

Remark 3.1 Application of these results to other equilibrium point is easily accomplished through coordinate transformations as explained in [Jlassi et al., 2012].

Remark 3.2 The decay rate of the tracking error can be adjusted by a suitable choice of $K$, which can be determined by pole placement techniques, optimal control etc.

\section{Application and simulation results}

\subsection{Penducobot example: main motivations for this choice}

A simple but demonstrative example of a Penducobot is discussed in this Section. A significant amount of research has amply treated this application but always within the context of robotic manipulation, as shown for example in [Fantoni et al., 2000] for the Pendubot manipulator or in [Matthew et al., 1999]) for the Acrobot example, where the objective of both works was to stabilize the mechanical system around an unstable equilibrium point. The control objectives of co-manipulation are different, as recalled in Section 2.

\subsection{Presentation of the Penducobot}

The current work focuses on illustrating the control strategy adopted through a single arm robot evolving in only one frame, depicted on Figure 6. Its architecture contains two revolute joints. The first one is active, located at point $O$ where a motor exerts a control torque $C_{m}$, and where $\theta$ is its measured position w.r.t. the fixed frame. The second is passive, located at point $\mathrm{A}$ and $\varphi$ measures its position w.r.t. the main arm. It is used to hang a pendulous load located at point $M$ and of mass $\mu$ through a massless bar, so that the proposed application is more akin to a practical case, like for example the one of a handling crane. The interaction point is located at point P, where the HO drives the robot and applies to it a force $\overrightarrow{F_{h}}$ as described in Subsection 3.1. The corresponding resulting torque, applied to the arm and exerted at the point $O$, is given by $C_{h}:=J(\theta)^{T} \overrightarrow{F_{h}}=L\left\|\overrightarrow{F_{h}}\right\|$ where $J(\theta)$ is the manipulator Jacobian. The joints at points $O$ and $A$ are assumed to be with viscous friction of friction factor $f_{O}$ and $f_{A}$ respectively, and where the friction torques are denoted $C_{f_{O}}$ and $C_{f_{A}}$ respectively. 


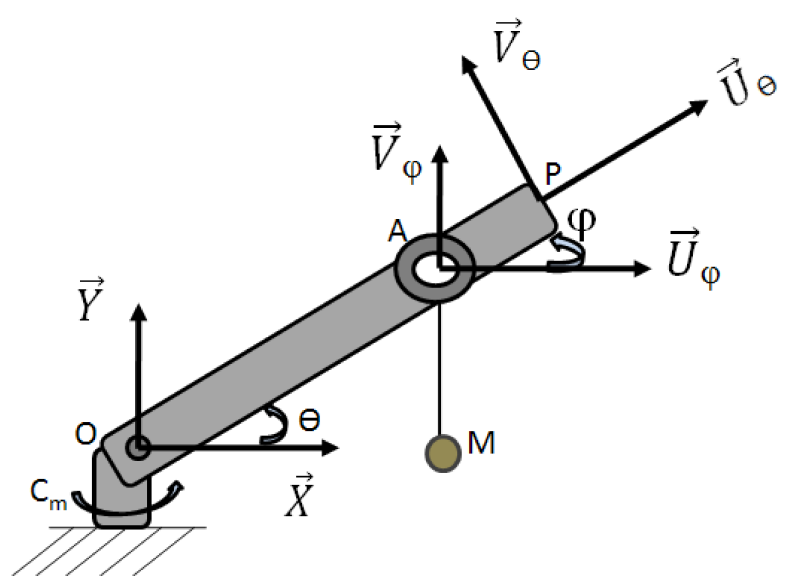

Figure 6: A rough sketch of the Penducobot.

Let $J_{\theta}:=m \frac{L^{2}}{3}+\mu l^{2}, J_{\alpha \theta}:=\mu l h, \gamma_{\theta}:=\left(m \frac{L}{2}+\mu l\right), \gamma_{\alpha}:=\mu h, \xi:=\cos (\theta-\alpha)$ and $\eta:=\sin (\theta-\alpha)$ where $\alpha:=\theta+\varphi+\frac{3 \pi}{2}$. The details of the motion equations (2) are given, in this case, by

$$
M(q):=\left[\begin{array}{cc}
J_{\theta} & J_{\alpha \theta} \xi \\
J_{\alpha \theta} \xi & J_{\alpha}
\end{array}\right], \quad C(q, \dot{q}):=\left[\begin{array}{cc}
0 & J_{\alpha \theta} \dot{\alpha} \eta \\
-J_{\alpha \theta} \dot{\theta} \eta & 0
\end{array}\right], \quad W_{f}:=\left[\begin{array}{c}
C_{f_{A}}+C_{f_{O}} \\
-C_{f_{A}}
\end{array}\right], \quad g(q):=\left[\begin{array}{c}
\gamma_{\theta} g \cos (\theta) \\
\gamma_{\alpha} g \cos (\alpha)
\end{array}\right]
$$

with $q^{a}:=\theta, q^{p}:=\varphi, q:=\left[\begin{array}{l}\theta \\ \alpha\end{array}\right], U:=\left[\begin{array}{l}\tau \\ 0\end{array}\right]$ and $\tau:=C_{m}+C_{h} \in \mathbb{R}$.

\subsection{OTG adaptation}

The OTG in Subsection 3.3 is adapted to the penducobot problem so that different force thresholds can be assigned to the rotation direction of the main arm, noted $f_{t h-}$ for the clockwise direction and $f_{t h+}$ for the counterclockwise one. Notice that the end-effector velocity norm is given here by $\|\dot{\vec{X}}\|=L|\dot{\theta}|$. Thus, generating a profile for $\|\dot{\vec{X}}\|$ or $|\dot{\theta}|$ is the same. Let $\omega_{0}$ be the cruise velocity of the IP desired velocity profile and $\tilde{\omega}_{0}=\operatorname{sign}\left\{\left\langle\overrightarrow{F_{h}}, \overrightarrow{V_{\theta}}\right\rangle\right\}\left|\omega_{0}\right|$ having the same sign as the scalar product $\left\langle\overrightarrow{F_{h}}, \overrightarrow{V_{\theta}}\right\rangle$ in order to take into account the rotation direction. Then, the velocity profile parameter $V_{0}$ of Paragraph b) corresponds here to $\tilde{\omega}_{0}$.

In order to illustrate the OTG behavior in a simple way, a sine function is assigned to $F_{h}\left(=\left\|\overrightarrow{F_{h}}\right\|\right)$. Figure 7 depicts the normalized human force $\widehat{F_{h}}(t):=\frac{F_{h}(t)}{\left\|F_{h}(t)\right\|_{\infty}}$ by the red curve, the trajectory progression of the endeffector velocity during the HRI, the normalized velocity profile (in blue) $\frac{\mathrm{d} \widehat{\theta_{d}}(t)}{\mathrm{dt}}=\frac{\dot{\theta}_{d}(t)}{\left\|\dot{\theta}_{d}(t)\right\|_{\infty}}$ and the normalized thresholds $f_{t h+}$ in dashed and $f_{t h-}$ in dot-dashed brown lines. One can observe the different phases following each other, identified by the terms RAMP, STOP and CRUISE:

- RAMP is used to designate both the rising and the falling phases,

- $\mathrm{STOP}$ is for the stopping phase,

- CRUISE is for the cruising phase.

Remark 4.1 The noises affecting the force measurement could lead the OTG to continuously switch between the different phases. This scenario could be avoided by the use of thresholds with hysteresis effect, in order to prevent small changes (corresponding to unwanted triggering events) from having any effect. 


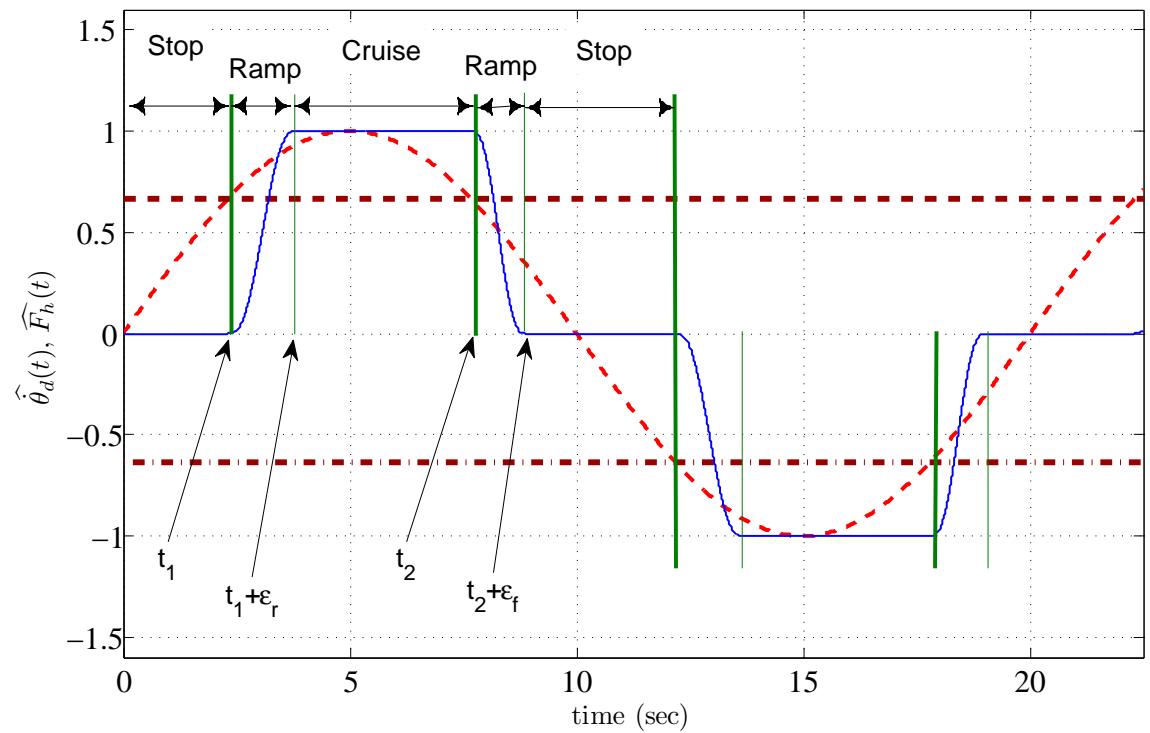

Figure 7: Desired trajectory for DOF $\dot{\theta}_{d}$ generated by the processing of force $F_{h}$.

\subsection{Linearization around an equilibrium point}

Let $x=\left[\begin{array}{llll}x_{1} & x_{2} & x_{3} & x_{4}\end{array}\right]^{T}:=\left[\begin{array}{llll}\theta & \alpha & \dot{\theta} & \dot{\alpha}\end{array}\right]^{T} \in \mathbb{R}^{4}$. By rewriting the motion equations in (2) with matrices of Subsection 4.2 into the corresponding state-space form as in (6), the set of computed equilibrium points is given by the couple $\left(x_{0}, U_{0}\right)$ where $x_{0}=\left[\begin{array}{llll}x_{0}^{1} & x_{0}^{2} & 0 & 0\end{array}\right]^{T}$, such that $\forall x_{0}^{1} \in\left[0,2 \pi\left[, x_{0}^{2}=\frac{\pi}{2}+k \pi \forall k \in \mathbb{Z}\right.\right.$, $U_{0}=\gamma_{\theta} g \cos \left(x_{0}^{1}\right)$. Given an arbitrary $x_{0}^{1}, U_{0}$ is then uniquely determined and there are two possible equilibrium positions for $x_{0}^{2}$. The computation of the Hessian of the potential energy function $E_{p}\left(x_{0}^{2}\right)$ allows to check that $\left(\left[\begin{array}{llll}x_{0}^{1} & x_{0}^{2} & 0 & 0\end{array}\right], U_{0}\right)$ where $\frac{3 \pi}{2}+2 k \pi \forall k \in \mathbb{Z}$, is a stable equilibrium point, corresponding to a "potential well". The linearization of the Penducobot model around the stable equilibrium point leads to a linear time invariant state-space model

$$
\begin{aligned}
\dot{z}(t) & =\mathbb{A} z(t)+\mathbb{B} v(t), \text { where } \\
\mathbb{A} & =\left[\begin{array}{cccc}
0 & 0 & 1 & 0 \\
0 & 0 & 0 & 1 \\
A_{31} & A_{32} & A_{33} & A_{34} \\
A_{41} & A_{42} & A_{43} & A_{44}
\end{array}\right], \mathbb{B}=\Delta\left(x_{0}\right)\left[\begin{array}{c}
0 \\
0 \\
J_{\alpha} \\
J_{\alpha \theta} \sin \left(x_{0}^{1}\right)
\end{array}\right],
\end{aligned}
$$

$$
\text { with } \begin{aligned}
\Delta\left(x_{0}\right) & =\left(J_{\alpha} J_{\theta}-J_{\alpha \theta}^{2} \sin \left(x_{0}^{2}-x_{0}^{1}\right)^{2}\right)^{-1}, \\
A_{31} & =\Delta\left(x_{0}\right)\left(J_{\alpha} \gamma_{\theta} g \sin \left(x_{0}^{1}\right)-4 \Delta\left(x_{0}\right) J_{\alpha} \gamma_{\theta} g \cos ^{2}\left(x_{0}^{1}\right) J_{\alpha \theta}^{2} \sin \left(x_{0}^{1}\right)\right), \\
A_{32} & =\Delta\left(x_{0}\right)\left(-g J_{\alpha \theta} \gamma_{\alpha} \sin \left(x_{0}^{1}\right)+4 \Delta\left(x_{0}\right) J_{\alpha} \gamma_{\theta} g \cos ^{2}\left(x_{0}^{1}\right) J_{\alpha \theta}^{2} \sin \left(x_{0}^{1}\right)\right), \\
A_{33} & =\Delta\left(x_{0}\right)\left(-\left(f_{A}+f_{O}\right) J_{\alpha}+f_{A} J_{\alpha \theta} \sin \left(x_{0}^{1}\right)\right), \\
A_{34} & =\Delta\left(x_{0}\right)\left(f_{A} J_{\alpha}-f_{A} J_{\alpha \theta} \sin \left(x_{0}^{1}\right)\right), \\
A_{41} & =\Delta\left(x_{0}\right)\left(J_{\alpha \theta} \gamma_{\theta} g\left(\sin ^{2}\left(x_{0}^{1}\right)-2 \cos ^{2}\left(x_{0}^{1}\right)\right)-4 \Delta\left(x_{0}\right) J_{\alpha \theta} \gamma_{\theta} g \cos ^{2}\left(x_{0}^{1}\right) \sin ^{2}\left(x_{0}^{1}\right)\right), \\
A_{42} & =\Delta\left(x_{0}\right)\left(2 J_{\alpha \theta} \gamma_{\theta} g \cos ^{2}\left(x_{0}^{1}\right)-g \gamma_{\alpha} J_{\theta}+4 \Delta\left(x_{0}\right) J_{\alpha \theta} \gamma_{\theta} g \cos ^{2}\left(x_{0}^{1}\right) \sin ^{2}\left(x_{0}^{1}\right)\right), \\
A_{43} & =\Delta\left(x_{0}\right)\left(f_{A} J_{\theta}-J_{\alpha \theta} \sin \left(x_{0}^{1}\right)\left(f_{A}+f_{O}\right)\right), \\
A_{44} & =\Delta\left(x_{0}\right)\left(-f_{A} J_{\theta}+f_{A} J_{\alpha \theta} \sin \left(x_{0}^{1}\right)\right) .
\end{aligned}
$$


Let $\mathcal{C}(\mathbb{A}, \mathbb{B})=\left[\begin{array}{llll}\mathbb{B} & \mathbb{A} B & \mathbb{A}^{2} \mathbb{B} & \mathbb{A}^{3} \mathbb{B}\end{array}\right]$ the controllability matrix. An analytical calculation gives the expression of its determinant:

$$
\Delta^{4}\left(x_{0}\right) g \gamma_{\alpha}\left(-\gamma_{\alpha} g J_{\alpha \theta}^{2} \sin ^{2}\left(x_{0}^{1}\right)+f_{A}^{2} J_{\alpha \theta} \sin \left(x_{0}^{1}\right)-f_{A}^{2} J_{\alpha}\right)
$$

Because of the presence of viscous friction within the joints,

$$
\operatorname{det}\{\mathcal{C}(\mathbb{A}, \mathbb{B})\}=0 \Rightarrow g M^{2} h^{2} l^{2} \sin \left(x_{0}^{1}\right)^{2}=f_{A}^{2}\left(l \sin \left(x_{0}^{1}\right)-h\right) \text {. }
$$

It is easy to check that if $f_{A}<2 M h \sqrt{h g}$, the system's controllability is verified regardless $x_{0}^{1}$. Otherwise, i.e.in the case of extremely high friction which is improbable for a realistic application, then the controllability property could be lost.

Remark 4.2 With no friction, one can check that the determinant is zero when $x_{0}^{1}= \pm k \pi, \forall k \in \mathbb{N}$. Thus, the system is controllable everywhere except when in horizontal position.

\subsection{Simulation results}

The following simulations were done using MATLAB/Simulink. The Penducobot parameters are $m=10[\mathrm{~kg}]$, $\mu=2[\mathrm{~kg}], l=4[\mathrm{~m}], L=5[\mathrm{~m}]$ and $h=0.5[\mathrm{~m}]$. The rise and fall times are set to $\varepsilon_{r}=0.6[s]$ and $\varepsilon_{f}=0.3[s]$. The positive threshold is set to $4.475[N]$ and the negative one to $-4.45[N]$. It corresponds to a torque threshold of roughly $22[\mathrm{Nm}]$. A viscous friction coefficient of $f_{O}=f_{A}=0.8[\mathrm{Nm} /(\mathrm{rad} / \mathrm{s})]$ is used for the friction model $W_{f_{m}}$. The initial positions of the robot arm and the rigid bar are $\theta(0)=\theta_{d}(0)=-45^{\circ}$ and $\phi(0)=45^{\circ}$ respectively.

The linearized system is stable in open loop and has two oscillating modes. The state feedback gain $K \in \mathbb{R}^{1 \times 4}$ of the control law (7) is computed so as to solve the Linear Quadratic control problem with the criterion

$$
J:=\int_{0}^{+\infty}(\Pi z(t))^{T} Q(\Pi z(t))+v(t)^{T} R v(t) d t
$$

where $\Pi$ is the matrix of coordinates change between variables $\{\theta, \alpha\}$ and $\{\theta, \varphi\}$. The $4 \times 4$ weighting matrix $Q \geq 0$, chosen bloc-diagonal to weight independently $\theta$ and $\varphi$, is customized to manage an acceptable trade-off between the tracking performances of DOFs $\theta, \varphi$ and their time derivatives. The scalar weighting $R>0$ allows to adjust the control effort in closed-loop. The proposed simulations were carried out for two cases of weighting parameters. The first was obtained by setting $R_{1}=10^{-9}$ and $Q_{1}=\operatorname{diag}\left(1,10^{-11}, 10^{-8}, 10^{-1}\right)$ in order to balance the tracking performances between DOF $\theta$ and DOF $\varphi$. The corresponding value of the state feedback gain is $K_{1}=\left[\begin{array}{llll}-3453.8 & 27116 & -34849 & -2979.6\end{array}\right]$ and the associated simulations are shown in Figure 8. The second was obtained by setting $R_{2}=10^{-9}$ and $Q_{2}=\operatorname{diag}\left(10^{4}, 10^{-6}, 10^{-3}, 10^{-3}\right)$ in order to emphasize the tracking performances of DOF $\theta$ with respect to $\operatorname{DOF} \varphi$. The corresponding value of the state feedback gain is $K_{2}=\left[\begin{array}{llll}3162100 & 25578 & 45.616 & 15.412\end{array}\right]$ and the associated simulations are shown in Figure 9. In each case of weighting parameters, one can note that tracking performances for the nonlinear system are quite similar to the linear system ones. Figure 8 shows that the tracking performance objective for the DOF $\theta$ has been worsened for the benefit of the one of $\operatorname{DOF} \varphi$, as expected by the choice of weighting parameters set in $Q_{1}$. On the other hand, Figure 9 reveals a degradation of the tracking performances for the DOF $\varphi$ when the weighting parameters emphasize the $\operatorname{DOF} \theta$ rather than $\varphi$, as set in $Q_{2}$. For this last, the state feedback gain contains higher values and causes more important oscillations in the trajectory tracking for DOF $\varphi$.

In conclusion, linearization technic appears to be quite suitable for dealing with the co-manipulation control problem formulated in this paper and applied to the Penducobot case. 

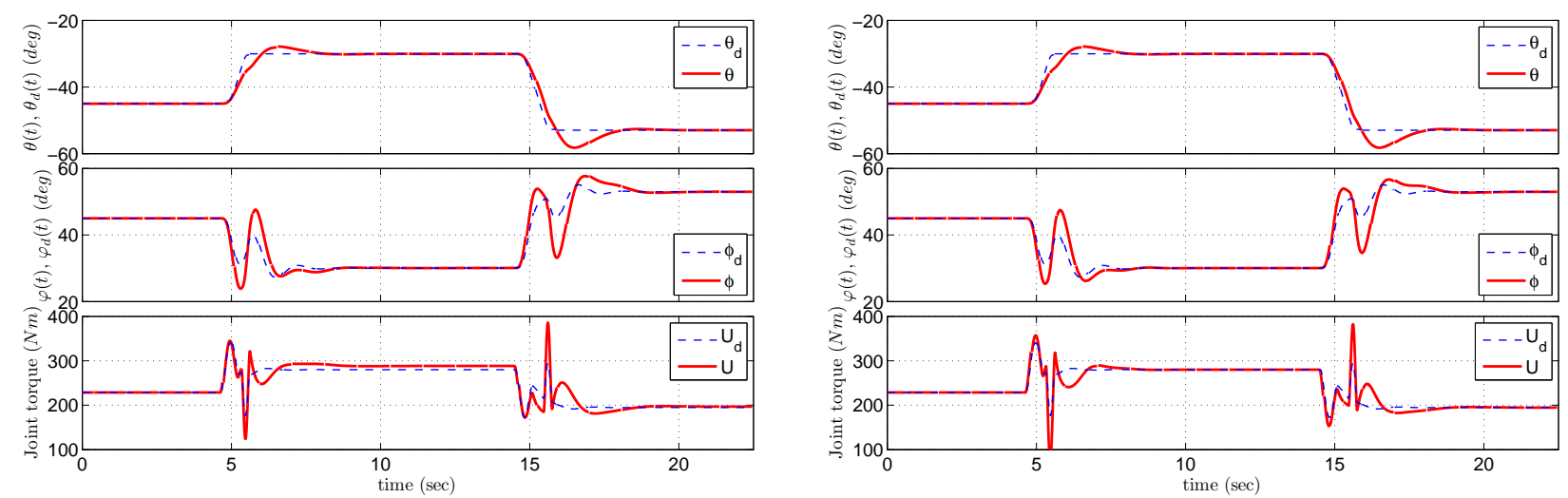

Figure 8: Trajectory tracking for the linearized system (left) and the nonlinear one (right) - Case of weighting parameters $\left(Q_{1}, R_{1}\right)$.
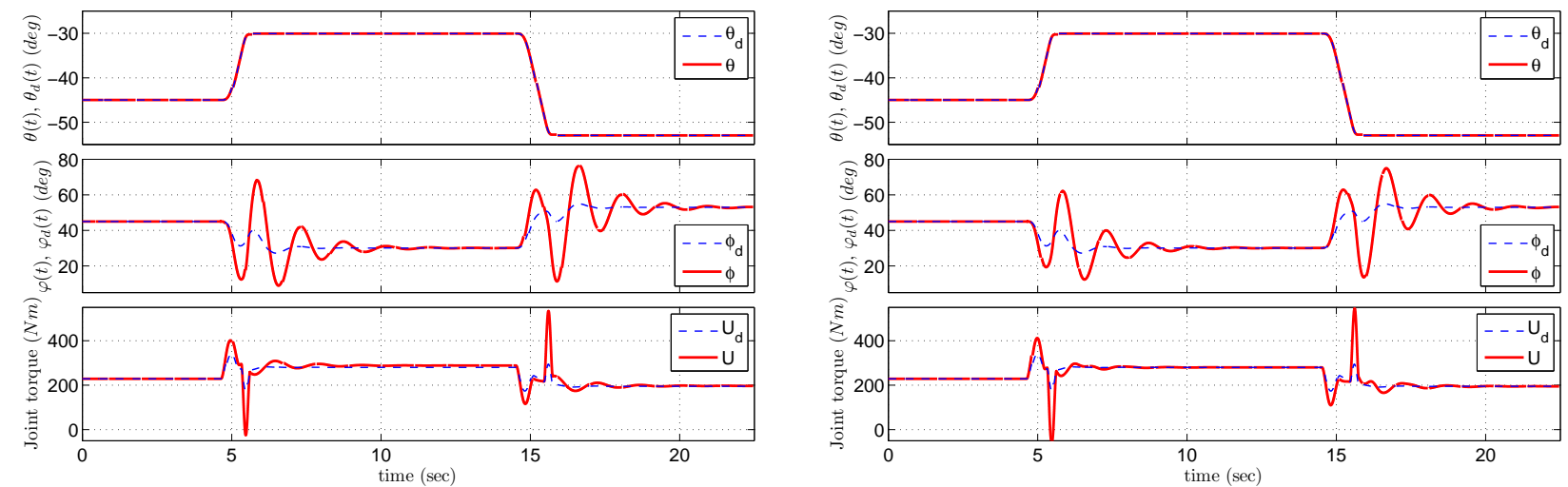

Figure 9: Trajectory tracking for the linearized system (left) and the nonlinear one (right) - Case of weighting parameters $\left(Q_{2}, R_{2}\right)$.

\section{Conclusions}

This paper addressed the co-manipulation problem for handling tasks through a specific OTG coupled with a motion feedback loop. The main issue consisted in designing a control strategy that allows a human to drive the robot end-effector anywhere he would like, at any time he would decide, according to the desired master-slave relationship depicted in Figure 1. The proposed approach mainly lies on an event-controlled OTG that delivers trajectories to track when the operator exerts a force which amplitude is greater than a given threshold of low intensity. The other part of this approach is based on the design of a kinematic feedback loop insuring good trajectory tracking and maintaining the immobility of the robot configuration when there is no interaction force. The resulting control strategy allowed illustrating the main idea that has been proposed, based on maintaining to the maximum collinearity between the end-effector velocity and the interaction force. By the choice of the penducobot application, we sought to apply our approach to a simple case, but similar to handling cranes encountered in industry.

\section{References}

[Akella et al., 1999] Akella, P., Peshkin, M., Colgate, E., Wannasuphoprasit, W., Nagesh, N., Wells, J., Holland, S., Pearson, T., and Peacock, B. (1999). Cobots for the automobile assembly line. In Robotics and Automation, Proc. of 1999 IEEE Int. Conf. on, volume 1, pages 728-733. 
[Buerger, 2005] Buerger, S. P. (2005). Stable, high-force, low-impedance robotic actuators for human-interactive machines. PhD thesis, Massachusetts Institute of Technology, Dept. of Mechanical Engineering.

[Colgate et al., 1996] Colgate, E., Peshkin, M., and Wannasuphoprasit, W. (1996). Cobots: Robots for collaboration with human operators. In Proc. of the ASME Dynamic Systems and Control Division, volume 58, pages $433-440$.

[Constantinescu and Croft, 2000] Constantinescu, D. and Croft, E. A. (2000). Smooth and time-optimal trajectory planning for industrial manipulators along specified paths. Journal of Robotic Systems, 17(5):233-249.

[Coron, 2007] Coron, J.-M. (2007). Control and Nonlinearity. American Mathematical Society.

[Craig, 1989] Craig, J. J. (1989). Introduction to Robotics. New York: Addison-Wesley.

[Duchaine and Gosselin, 2011] Duchaine, V. and Gosselin, C. (2011). Unified robot control scheme for cooperative motion, autonomous motion and contact reaction. J. of Robotics and Mechatronics, 23(4).

[Fantoni et al., 2000] Fantoni, I., Lozano, R., and Spong, M. (2000). Energy based control of the pendubot. Automatic Control, IEEE Trans. on, 45(4):725-729.

[Flash and Hogans, 1985] Flash, T. and Hogans, N. (1985). The coordination of arm movements an experimentally confirmed mathematical model. The Journal of Neurosciences, 5(7):1688-1703.

[Ikeura et al., 1994] Ikeura, R., Monden, H., and Inooka, H. (1994). Cooperative motion control of a robot and a human. In Robot and Human Communication, 1994. RO-MAN '94 Nagoya, Proceedings., 3rd IEEE International Workshop on, pages 112-117.

[Itoh et al., 2000] Itoh, T., Kosuge, K., and Fukuda, T. (2000). Human-machine cooperative telemanipulation with motion and force scaling using task-oriented virtual tool dynamics. Robotics and Automation, IEEE Trans. on, 16(5):505-516.

[Jlassi et al., 2012] Jlassi, S., Tliba, S., and Chitour, Y. (2012). On human-robot co-manipulation for handling tasks: Modeling and control strategy. In Proc. of the 10th International IFAC Symposium on Robot Control (SYROCO 2012), page 710, Dubrovnik, Croatie. International Federation of Automatic Control.

[Kazerooni and Guo, 1993] Kazerooni, H. and Guo, J. (1993). Human extenders. Journ. of Dyn. Syst., Meas. and Control, 115:281-290.

[Kröger, 2010] Kröger, T. (2010). On-Line Trajectory Generation in Robotic Systems Basic Concepts for Instantaneous Reactions to Unforeseen (Sensor) Events. Springer-Verlag Berlin Heidelberg.

[Lamy et al., 2010] Lamy, X., Colledani, F., Geffard, F., Measson, Y., and Morel, G. (2010). Human force amplification with industrial robot: Study of dynamic limitations. In Intelligent Robots and Systems (IROS), 2010 IEEE/RSJ Int. Conf. on, pages 2487-2494.

[Lawitzky et al., 2010] Lawitzky, M., Mörtl, A., and Hirche, S. (2010). Load sharing in human-robot cooperative manipulation. In RO-MAN, Proc. of 2010 IEEE, pages 185-191.

[Matthew et al., 1999] Matthew, D., Berkemeier, S., and Fearing (1999). Tracking fast inverted trajectories of the underactuated acrobot. Robotics and Automation, IEEE Trans. on, 15(4).

[Miyhoshi and Murata, 2000] Miyhoshi, T. and Murata, A. (2000). Chaotic characteristic in human hand movement. In Robot and Human Interactive Communication, 2000. (RO-MAN 2000). Proceedings. 9th IEEE International Workshop on, pages 194-199. 
[Piazzi and Visioli, 2000] Piazzi, A. and Visioli, A. (2000). Global minimum-jerk trajectory planning of robot manipulators. Industrial Electronics, IEEE Transactions on, 47(1):140-149.

[Santis et al., 2008] Santis, A. D., Siciliano, B., Luca, A. D., and Bicchi, A. (2008). An atlas of physical humanrobot interaction. Mechanism and Machine Theory, 43(3):253-270.

[Spong et al., 2006] Spong, M. W., Hutchinson, S., and Vidyasagar, M. (2006). Robot modeling and control. John Wiley and Sons, Inc.

[Tee et al., 2010] Tee, K. P., Franklin, D. W., Kawato, M., Milner, T. E., and Burdet, E. (2010). Concurrent adaptation of force and impedance in the redundant muscle system. Biological cybernetics, 102(1):31-44.

[Wannasuphoprasit et al., 1997] Wannasuphoprasit, W., Gillespie, R., Colgate, J., and Peshkin, M. (1997). Cobot control. In Robotics and Automation, Proc. of 1997 IEEE Int. Conf. on, volume 4, pages 3571-3576. 